\title{
A 12-week primary prevention programme and its effect on health outcomes (the Sweet Hearts biokinetics pilot study)
}

\author{
R W Evans, ${ }^{1}$ MPhil; D J Hume, ${ }^{1} \mathrm{PhD}$; M H Noorbhai, ${ }^{2} \mathrm{PhD}$; HG L \\ Rauch, ${ }^{1} \mathrm{PhD}$; N van der Schyff, ${ }^{3 \mathrm{MD}}$; E V Lambert, ${ }^{1} \mathrm{PhD}$ \\ ${ }^{1}$ Division of Exercise Science and Sports Medicine, Department of Human \\ Biology, Faculty of Health Sciences, University of Cape Town, Newlands, \\ 7725, South Africa \\ ${ }^{2}$ Department of Sport Management, Faculty of Business and Management \\ Sciences, Cape Peninsula University of Technology, Mowbray, 7925, South \\ Africa \\ ${ }^{3}$ Victoria Hospital, Alphen Road, Wynberg, 7780, Cape Town, South Africa
}

Corresponding author: RW Evans (RobertEvansSA@gmail.com)

Background: The prevalence of non-communicable diseases (NCDs) and physical inactivity are concerning within the South African population. To address these concerns, the 'Strategic Plan for Prevention and Control of NCDs 2013-2017' was developed. In response to this plan, a 12-week pilot biokinetics community health programme, Sweet Hearts, was initiated.

Methods: This study is a prospective pilot study evaluating the feasibility and effectiveness of the intervention. Twentyfive individuals participated in the intervention. Ten participants performed a battery of physiological tests pre and post intervention and 5 participants completed an email-based survey post intervention. The setting of the study was Tramway Football Club, Southfield, Cape Town, South Africa. The Sweet Hearts intervention was designed to promote physical activity and healthy nutritional habits in those who participated. A total of 27 exercise sessions consisting of cardiovascular, resistance and flexibility training were conducted. Brief-behavioural counselling was integrated into exercise sessions.

Results: The intervention group had a high attrition rate with $>50 \%$ of participants not presenting for post-intervention testing. Results were evident despite a limited sample size. There were significant improvements in health outcome measures among participants who did attend all testing sessions. These improvements included: an increase in Global Physical Activity Questionnaire (GPAQ) score $(p=0.03), 12$ minute walk distance $(p=0.01)$, sit-to-stand test repetitions ( $p$ $=0.001)$, and a decrease in waist circumference $(p=0.01)$. Improvements were also noted in self-reported eating restraint ( $p=0.03)$. Five main themes were structured into post intervention surveys: 1 ) enjoyment of the intervention, 2) benefits of the intervention, 3) obstacles affecting adherence, 4) future improvements to the intervention, and 5) state of non-communicable diseases in South Africa.

Conclusion: The results of the Sweet Hearts intervention demonstrate the difficulty and importance of maintaining adherence to a community health intervention. The favourable results of the small sample size demonstrate the potential benefit of biokinetics-based programmes in the public health sector; and provide proof of concept for the dedication of resources towards health promotion within a community setting.

Keywords: non-communicable diseases, physical inactivity, public health, community intervention, adherence S Afr J Sports Med 2017;29:1-7. DOI: 10.17159/2078-516X/2017/v29i0a1346

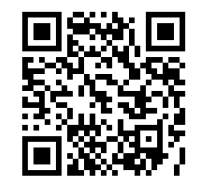

Non-communicable diseases (NCDs) are noninfectious, chronic conditions that are typically lifestyle-related, and are generally treatable via health promotion and structured behaviour modification. ${ }^{[1]}$ NCDs typically progress at a slow rate and include (but are not limited to) cardiovascular disease, various types of cancer, chronic respiratory diseases, metabolic dysfunction, and type 2 diabetes mellitus. The predominant causes of mortality globally are from NCDs. ${ }^{[2]}$

Physical inactivity is recognised internationally as a major independent and modifiable risk factor contributing to the worldwide rise in NCDs. Currently more than $50 \%$ of adults within South Africa are physically inactive. ${ }^{[2]}$ Based on the South African Demographic and Health Survey (SADHS) data collected in 1998, Bradshaw et al. identified that an alarming number of NCDs are attributable to physical inactivity among South Africans.[1] For example, in this country, physical inactivity is implicated in $30 \%$ of patients with ischaemic heart disease and in $20 \%$ of those presenting with type 2 diabetes mellitus.

The South African Summit on the Prevention and Control of NCDs, held in 2011, reached a formal consensus identifying the need for an intensified national plan stressing prevention, early detection, behavioural change and the nationwide treatment of NCDs. Emerging from this meeting, the Strategic Plan for the Prevention and Control of Non-Communicable Diseases 20132017 - developed by the South African Department of Health (DoH) - outlines three main public health strategies: 1) prevent NCDs and promote health and wellness at population, community and individual levels; 2) improve control of NCDs through the strengthening and reform of health systems; and 3) the monitoring of NCDs and their main risk factors, as well as conducting innovative research. ${ }^{[2]}$ In order to assist with the attainment of the DoH's goals, a 12-week pilot biokinetics primary prevention programme - the Sweet Hearts study - was initiated.

The problem of physical inactivity is further compounded by the fact that half or less of individuals fail to adhere to exercise programmes over the long-term or before significant health benefits can be realised. ${ }^{[3]}$ Notably, evidence suggests that people from low-income groups, such as those reliant on the public health sector, are more difficult to identify and successfully recruit for guided lifestyle modification. ${ }^{[3]}$ Examples of non-adherence are well documented by Scarinci et al. ${ }^{4]}$ where $45 \%$ of participants were lost to follow-up. As such, strategies to promote adherence must be incorporated into the initial design of physical activity interventions if they are to have a meaningful impact on the people they serve.

Numerous lifestyle modification programmes negate the social, political, cultural and economic forces that shape individual choices in their daily lives. A potential solution for this is the social-ecological model, which incorporates several lateral influences and acknowledges the value and complexities of creating an environment which promotes physical activity. ${ }^{[5]}$ The social-ecological model was thus implemented during the conceptualisation and execution of this project, the findings of 
which are discussed in this context.

In the present study, a pilot physical activity-based community health intervention programme was implemented. The intervention was developed as a response to the call by Evans et al. ${ }^{[6]}$ for the inclusion of biokinetics into the South African public health system and furthermore, to act upon the recommendations set out in the Strategic Plan for the Prevention and Control of NCDs 2013-2017. The primary goals of the intervention were to (a) increase the physical activity levels of participants and (b) promote improved lifestyle choices including healthy eating and stress reduction. In addition, participants completed a post-intervention survey to capture qualitative data so as to elucidate subjective experiences throughout the intervention.

\section{Methods}

\section{Study design}

In this prospective pilot study, participants were enrolled in a 12-week physical activity-based programme. They were assessed at baseline and at the end of the 12-week programme. The testing protocol included health screening, aerobic and muscular endurance, various health-related questionnaires and a semi-structured survey retrospectively evaluating the subjective experiences of the programme. The study was conducted with approval from the Faculty of Health Sciences Human Research Ethics Committee (HREC REF 214:2012; 467:2015), University of Cape Town, South Africa.

Tramway Football Club in Southfield, Cape Town, served as the venue for the study. Southfield is one of Cape Town's Southern Suburbs. NCDs account for a total of $31 \%$ of yearsof-life-lost in the Southern Suburb's populations. ${ }^{[7]}$ The large prevalence of NCDs in these populations made it an appropriate setting for a community lifestyle intervention.

\section{Participants}

Twenty-five participants (22 females, 3 males) were recruited from the community into the 12 -week intervention via radio announcements, flyers and emails. Participants were excluded on the grounds of orthopaedic injury presentation, or possessing $\geq$ two risk factors ${ }^{*}$ based on the American College of Sports Medicine (ACSM) risk stratification categories for cardiovascular disease. ${ }^{[8]}$

\section{Measurements}

Testing was conducted by qualified and intern biokineticists with the assistance of the Biokinetic Humanitarian Project (BHP). ${ }^{[9]}$

\section{Questionnaires}

On the first day of testing, participants completed an informed consent form and ACSM pre-participation

\footnotetext{
* A review of risk assessment and physical activity clearance by Thomas et al. [16] provides evidence that ordinarily 'high-risk' individuals ( $\geq 2$ ACSM risk factors) who are medically stable, partake in physical activity, and have adequate physical ability, should participate in medically-supervised physical activity interventions of low-to-moderate risk. Based on these recommendations, the on-site
}

questionnaire designed to identify any factors that indicate a risk of participation in exercise. Participants then completed self-report questionnaires ${ }^{1^{* *}}$ designed to measure their subjectively quantified physical activity level (Global Physical Activity Questionnaire), demographics, eating habits (ThreeFactor Eating Questionnaire), tobacco use and resilience (CDRISC). The Three-Factor Eating Questionnaire (TFEQ) is a 51item survey that measures three dimensions of human eating behaviour: cognitive eating restraint (the tendency to restrict food intake to control body weight), disinhibition (a sporadic loss of dietary control in the form of increased frequency and/or volume of food intake), and hunger (a measure of whether appetite primarily drives food intake, and the extent to which the individual engages in emotional eating). Higher scores indicate greater degrees of cognitive restraint, uncontrolled eating, or hunger. The TFEQ and its modified versions have been shown to be valid measures of these habitual eating behaviours in healthy populations. ${ }^{[10]}$

Brief behavioural counselling was performed with participants to establish individualised and achievable goals whilst participating in the intervention. Qualitative measurements were conducted upon completion of the 12-week programme which involved a self-developed open-ended survey. This structure was used to ensure all pertinent topics were covered, whilst also further allowing the participant to discuss a wide range of issues. A purposive sampling technique was used to select participants for the email-based survey. Of the total of ten participants who completed the intervention, five agreed to complete the survey.

\section{Anthropometry and health screening}

Participants' stature (Seca stadiometer, Seca, USA), body mass (Clover scale, model TCS-A300, South Africa), waist circumference (standard non-elastic tape measure), blood pressure (manual), blood glucose (Accu-Chek, Roche Diagnostics, USA), resting heart rate (manual) and the thickness of seven skinfolds (Harpenden caliper, Holtain Ltd, Bryberian, Crymmych, Pembrokeshire) were then measured.

\section{Aerobic capacity and muscular endurance}

Participants then performed a one-minute lower body muscle endurance test and a 12-minute walking test ${ }^{[11]}$ where they were encouraged to walk as far as possible in 12 minutes. The lower body muscle endurance test involved sitting to standing from a chair as many times as possible in 60 seconds. This testing protocol was also repeated after the 12-week intervention.

\section{Intervention}

A total of 27 exercise sessions, 60 minutes in duration, were conducted during the 12-week intervention. Sessions were held on Monday and Wednesday evenings. All sessions were

medical doctor assessed those who still wished to participate but would ordinarily be excluded according to the ACSM criteria. Participants were included if it was deemed the benefits they stood to gain from the study outweighed the risks of participation. No adverse events occurred during the course of the intervention.

** References pertaining to questionnaires used are available on request from the corresponding author. 
planned and conducted by intern biokineticists completing their final year of practical training and were supervised by two qualified biokineticists.

Each session progressed from low to moderate intensity (approximately $40-70 \% \quad \mathrm{VO}_{2} \mathrm{max}$ ) and contained a combination of strength, cardiovascular and flexibility training. Exercises were conducted on an open field and basic equipment, such as cones, skipping ropes, elastic resistance bands and medicine balls, were used. Examples of exercises prescribed included push-ups, squats, elastic rows, pelvic bridges, repeat sprints, agility drills, medicine ball throws, hamstring stretches etc.

Brief behavioural counselling was conducted during warm up and cool-down periods. The counselling addressed pertinent lifestyle issues, such as physical inactivity, smoking and eating behaviours. Intern biokineticists were trained in basic life support and a medical doctor was either on-site or on emergency call.

\section{Statistical analysis}

The Statistica 12 software package was used for all analyses. Quantitative analysis was performed on the ten participants completing the intervention while qualitative analysis was performed on the five participants who agreed to partake in the survey. A goodness-of-fit model was used to confirm that data were parametric. Descriptive statistics were performed and reported as means \pm standard deviations. Inferential statistics (student $t$-tests) were utilised to detect differences between participants at baseline and post-intervention. The alpha level was set at $p<0.05$. Survey results were manually colour-coded for content in order to determine common themes.

\section{Results}

\section{Quantitative results}

Twenty-five individuals were initially enrolled in the study with an average attendance of $56 \%$ across all sessions. Of these, ten participants (all females) made themselves available for post-testing with an average attendance of $72 \%$ (Table 1 ); a minimum attendance rate of $60 \%$ was required to be included in the final analysis. Variables at baseline were equivalent in those presenting for post-testing versus those who did not; except for a significantly higher resting heart rate $(\mathrm{p}=0.01)$ and significantly lower resting breathing rate $(\mathrm{p}=$ $0.04)$ in those who could not attend the post-testing session.

Table 1. General characteristics of participants

\begin{tabular}{lc}
\hline \multicolumn{1}{c}{ Variables } & Value $(\mathbf{n}=\mathbf{1 0})$ \\
\hline Age $($ years $)$ & $33 \pm 11.5$ \\
Height $(\mathrm{cm})$ & $162.3 \pm 5.5$ \\
Body mass index $(\mathrm{kg} / \mathrm{m} 2)$ & $29.4 \pm 8.5$ \\
Income group & $5.3 \pm 2.2$ \\
Smokers $(\%)$ & $40 \%$ \\
Medical aid members (\%) & $60 \%$ \\
\hline Values reported as mean \pm sd & \\
* Income groups: $1=$ No income; $2=R 1-R 2499 ;$ \\
$3=$ R2500-R4999; $4=R 5000-R 7499 ; 5=R 7500-R 9999 ;$ \\
$6=R 10$ 000-R15000; $7=R 15000$ or more
\end{tabular}

Significant changes occurred in the following basic physiological variables $(p \leq 0.05)$ : an increase in the Global Physical Activity Questionnaire (GPAQ) score from $689 \pm 791$ to $2760 \pm 2523$ MET-minutes/week $\left(\mathrm{t}_{(8)}=2.57, \mathrm{p}=0.03\right)$, an increase in 12-minute walk distance from $1360 \pm 353$ to $1565 \pm$ 220 meters $\left(\mathrm{t}_{(9)}=3.15, \mathrm{p}=0.01\right)$, an increase in the number of sitto-stands from $35.8 \pm 6.3$ to $42.5 \pm 3.4$ repetitions $\left(t_{(9)}=4.61, p=\right.$ $0.001)$, and a decrease in waist circumference from $85.9 \pm 15.4$ to $80.8 \pm 11.4 \mathrm{~cm}\left(\mathrm{t}_{(9)}=-3.2, \mathrm{p}=0.01\right)$. A summary of changes in basic physiological variables is contained in Table 2 below.

Table 2. Basic physiological changes over 12-week intervention period

\begin{tabular}{lccc}
\multicolumn{1}{c}{ Variables } & $\begin{array}{c}\text { Baseline } \\
(\mathbf{n}=\mathbf{1 0})\end{array}$ & $\begin{array}{c}\text { Post- } \\
\text { intervention } \\
(\mathbf{n}=\mathbf{1 0})\end{array}$ & $\mathbf{p}$ \\
\hline Body mass index (kg/m2) & $29.4 \pm 8.5$ & $29 \pm 8.6$ & 0.26 \\
Body fat (\%) & $34 \pm 4$ & $35 \pm 4$. & 0.53 \\
Waist circumference (cm) & $85.9 \pm 15.4$ & $80.8 \pm 11.4$ & $0.01^{* *}$ \\
Systolic blood pressure & $117 \pm 8$ & $105 \pm 37$ & 0.31 \\
(mmHg) & & & \\
Diastolic blood pressure & $76 \pm 8$ & $78 \pm 10$ & 0.49 \\
(mmHg) & & & \\
Resting heart rate & $80 \pm 16$ & $77 \pm 11$ & 0.30 \\
(beats.min-1) & $5.4 \pm 0.9$ & $5.3 \pm 0.38$ & 0.69 \\
Blood glucose (m.mol.l-1) & $689 \pm 791$ & $2760 \pm 2523$ & $0.03^{*}$ \\
$\begin{array}{l}\text { GPAQ Score } \\
\text { (MET-minutes/week) }\end{array}$ & & & \\
12-minute walk test (m) & $1360 \pm 353$ & $1565 \pm 220$ & $0.01^{* *}$ \\
Sit-to-stand test (repetitions) & $36 \pm 6$ & $43 \pm 3$ & $0.001^{* *}$ \\
CD-RISC score & $73.8 \pm 10.6$ & $75 \pm 8.2$ & 0.66 \\
\hline
\end{tabular}

Values reported as mean $\pm s d$

* Significant differences between baseline and post-intervention $(p<0.05)$;

** Significant differences between baseline and post-intervention $(p<0.02)$

Scores on the Three-Factor Eating Questionnaire showed favourable changes across dietary restraint, disinhibition and hunger scores (Table 3). However, only the increase in restraint reached a level of significance $\left(\mathrm{t}_{(9)}=2.51, \mathrm{p}=0.03\right)$.

Table 3. Three-Factor Eating Questionnaire changes over 12-week intervention period

\begin{tabular}{llll}
\hline \multicolumn{1}{c}{ Variables } & $\begin{array}{c}\text { Baseline } \\
(\mathbf{n}=\mathbf{1 0})\end{array}$ & $\begin{array}{c}\text { Post- } \\
\text { intervention } \\
(\mathbf{n}=\mathbf{1 0})\end{array}$ & $\mathbf{p}$ \\
\hline Dietary restraint (score) & $7.2 \pm 5.2$ & $9.9 \pm 5.9$ & $0.03^{*}$ \\
Disinhibition (score) & $7.6 \pm 4$ & $5.7 \pm 3.1$ & 0.15 \\
Hunger (score) & $5.7 \pm 4$ & $4.5 \pm 3.4$ & 0.31 \\
\hline
\end{tabular}

Values reported as mean \pm sd

* Significant differences between baseline and post-intervention $(p<0.05)$;

\section{Qualitative results}

Five primary themes were structured into the survey: 1) enjoyment of the intervention 2) benefits of the intervention 3) obstacles affecting adherence 4) future improvements to the intervention 5) state of NCDs in South Africa. Quotations to support the analysis are identified by a code, indicating their source respondent $(\mathrm{RD})$.

\section{Enjoyment of the intervention}

Respondents identified fun and pleasure whilst being physically active as major motivators for continued adherence 
to the intervention. Exercise sessions were light-hearted, with camaraderie between participants. Variation in exercise drills ensured that participants were not partaking in monotonous activities.

"I love exercising, so this was my main motivation. (RD1)"

"I enjoyed the varied nature of workouts; I was never bored. (RD2)"

Group dynamics played an important role in the success of the intervention. Participants enrolled as strangers but formed friendships during the intervention. The group setting allowed participants to work at their own pace whilst still being encouraged to challenge themselves. A family (mother and two daughters) was able to spend more time together as a result of participating in the intervention.

"I felt encouraged, supported and motivated by other participants and so worked harder than I might otherwise have in a solo endeavour; like gym (RD2)"

"I love exercising! My mom and my sister don't. Glad we found something that they would enjoy as well. (RD3)"

"I felt the ..... group dynamic, which I knew I would be more accountable for and to, were draw cards. (RD2)"

Respondents discussed the satisfaction they gained from challenging themselves. Muscle aches in the days after training were not seen as negative but rather a sign that they had sufficiently worked the body and overcame perceptions of fatigue.

"Most people don't enjoy the muscle pains after a good workout. I do. Just like the saying goes, 'no pain, no gain!' (RD3)"

The intervention was considered convenient for participants. The times of training were well suited and coincided with some of the participants' children's soccer practices.

"I couldn't drop my son off [at soccer practice] and not attend a session! (RD4)"

\section{Benefits of the intervention}

Respondents mentioned the physiological changes brought about by an increase in physical activity. The changes discussed include an increase in fitness, muscle tone, decreased body mass, increased awareness of dietary intake and assistance with injuries.

"I not only lost weight, but I gained muscle and became more fit. And eating unhealthy didn't feel right anymore, so we just didn't (RD4)"

The structured, scientific focus of the intervention assisted participants in establishing safe exercise routines. The intervention also taught participants the value of becoming more responsible for their own health.

"Establishing the routine also helped me to become more accountable to myself by requiring me to proactively set up boundaries: my family and employer knew that on the days and during the time of training I was not available, which freed me up to concentrate on my fitness goals. (RD2)"

"I learnt the correct way to do actual exercises to prevent injuries. (RD4)"

The difficulties of exercise sessions were tailored to each individual. Instructors were flexible in their exercise prescription, whilst providing encouragement or empathy when appropriate.

"The positive feedback from trainers was also a plus - there was always encouragement to work beyond perceived limitations but empathy when any of us were genuinely taking strain. (RD2)"

\section{Obstacles affecting adherence}

The intervention was conducted throughout the Cape Town winter (cold and rainy months) and hence poor weather was an obstacle to participation. Only one exercise session was officially cancelled due to heavy rain; however, cold and wet weather did affect adherence.

"It was difficult to motivate myself to train when it was wet and rainy. $(R D 5) "$

Family and work commitments reduced the time available to respondents to participate in the intervention. Four of the five participants surveyed had children and four of the five were employed.

"Only difficulty with attending sessions was when they clashed with scheduled meetings at work. (RD5)"

"I have a spouse and 4 kids. (RD4)"

\section{The state of non-communicable diseases (NCDs) in South Africa}

Respondents from this study were generally aware of the negative impact that NCDs such as obesity, diabetes and heart disease have on the country. A career-focused instead of a health-focused society that promotes the consumption of fast food was acknowledged. These societal influences, combined with modern technology that diminishes habitual physical activity levels, were seen to be one of the factors fuelling the rapid rise in NCDs.

"In my extended family we're constantly griping about how although we're relatively intelligent and enjoy successful careers, we can't shake off the weight and all of the lifestyle related diseases that are attendant to it. (RD2)"

The study's respondents no longer regarded access to equipment as a barrier to physical activity. The intervention was able to conduct a wide variety of exercise drills with minimal equipment. Participants also identified that changing one's lifestyle is not a solitary effort but that support from peers is crucial in modifying dietary and activity behaviours.

"I was struck by how few resources we needed for the programme. We are socialised to believe that in the absence of contraptions and equipment we are unable to exercise. So the greatest benefit of having access to a biokineticist would be learning how to effectively and naturally get one's heart rate up and muscles working. (RD2)"

"The fact that someone was actually waiting for me is what got me there (RD3)"

Poor education and low socio-economic status were mentioned as factors associated with the rise in NCDs. It was perceived that many individuals in South Africa have little knowledge about the importance of exercise or nutrition and their effect on health. With poor awareness of the problem at hand and insufficient income to make healthy lifestyle choices, it is likely the burden of NCDs will continue to rise.

"More focus needs to be placed on areas where knowledge and 
information about health, exercise and diet are not freely available. (RD3)"

\section{Discussion}

\section{Quantitative}

The aim of this study was to (a) increase the physical activity levels of participants and (b) promote improved lifestyle choices, including healthy eating and stress reduction. Over the duration of the 12-week intervention, the primary findings of the study were in the following physiological variables $(\mathrm{p} \leq$ 0.05): an increase in the Global Physical Activity Questionnaire (GPAQ) scores, self-reported eating restraints, 12-minute walks, sit-to-stand tests and a decrease in waist circumference. Poor adherence and the resulting low sample size means no solid conclusions can be drawn from these results. It is also important to consider that a social desirability bias may have influenced the self-report measures. Despite these limitations, these findings may, however, assist in generating hypotheses for future research.

With the addition of two regular physical activity sessions per week, participants who were largely sedentary at baseline increased their physical activity levels (GPAQ score) during the intervention. Aerobic capacity and lower body muscular endurance, measured through the 12-minute walk and sit-tostand tests respectively, also significantly increased during the intervention.

Koster et al. 12] examined the association between waist circumference and mortality among 154776 men and 90757 women in the NIH-AARP Diet and Health Study. In comparison to participants with a normal BMI and waist circumference, those in the normal BMI group with a large waist circumference (men $>102 \mathrm{~cm}$; women $>88 \mathrm{~cm}$ ) had an approximately $20 \%$ higher mortality risk. This finding supports the fact that an elevated waist circumference is an independent risk factor for all-cause mortality. A statistically significant average decrease of five $\mathrm{cm}$ in participants' waist circumference may confer health benefits and lower the risk of all-cause mortality.

Within this study, participants' level of restraint regarding eating behaviours improved on the Three-Factor Eating Questionnaire. In a cross-sectional study of 529 middle-aged adults and 358 teenagers and young adults, cognitive restraint was positively associated in eating healthy food groups, such as green vegetables, and negatively associated with French fries (chips) and sugar. ${ }^{[10]}$ Therefore the increase in cognitive restraint observed over the course of the intervention may have resulted in improved dietary choices amongst participants.

\section{Qualitative}

\section{Adherence}

The subjective experience of participants has been captured in the qualitative results section above. The following qualitative discussion will focus upon adherence, as it is a crucial component contributing to the success of a community-health intervention. ${ }^{[3]}$ Community-health interventions assist in promoting healthy behaviours; however, they are only effective if adhered to. Modern environmental and personal circumstances often obstruct healthy lifestyle choices. Determinants of adherence to a community-health intervention are complex. Under the paradigm of the social ecological model, these determinants are a product of both personal and environmental characteristics. ${ }^{[5]}$

\section{Personal characteristics}

Personal characteristics include factors such as motivation, current health, desired appearance, enjoyment, social interaction, stress relief, challenge, skill development, achievement and personal satisfaction. Respondents saw improvements in personal benefits from attending the exercise sessions. These subjective benefits included increased fitness, healthier eating habits and improved body composition.

No personal characteristics were seen as barriers to participation as the participants surveyed had positive attitudes towards physical activity. This finding is supported by Mccready and Long ${ }^{[13]}$ who found that participants with a more positive attitude toward exercising to reduce stress and tension, tended to have a higher attendance percentage.

Throughout the duration of the intervention, participants steadily progressed in the difficulty of the exercises performed, thus increasing their self-efficacy with regard to physical activity. Previous research has demonstrated that with continued exercise participation, self-efficacy increases and adherence increases concurrently. ${ }^{[14]}$ A prior history of physical activity is also beneficial as it positively influences physical activity behaviour during an intervention by promoting and shaping self-efficacy for exercise. ${ }^{[14]}$

\section{Social environmental characteristics}

Social environmental characteristics include factors such as demographics, work and family commitments, education and exercise group dynamics. Behaviour change can only occur when both the individual and their social environment support that change. Social support within a group exercise setting increases adherence to physical activity due to the 'bonding' experience of setting and reaching goals together. ${ }^{[14]}$ For many of the participants, being part of a group was integral to their adherence and the camaraderie with fellow participants helped them maintain their physical activity levels.

Although time availability is a personal resource, lack of time is typically attributed to external factors. ${ }^{[15]}$ Family and work commitments are often prioritised over one's own health, leading to reductions in physical activity. This is supported by White et al. ${ }^{[14]}$ who demonstrated that becoming a parent is associated with reductions in physical activity for mothers. Time availability was the greatest obstacle to adherence during this intervention. Many participants prioritised other daily tasks over their participation.

Participants gained the most enjoyment from the social environmental characteristics of the intervention. These characteristics included the positive group dynamics, convenient location and time as well as the structured scientific approach. Poor weather was the only environmental constraint mentioned in the surveys. In future interventions, this barrier can be addressed by acquiring the use of an indoor facility.

\section{Reflexivity}

Reflexivity refers to the recognition of the influence a researcher 
brings to the research process. As researchers within the physical activity and public health fields, the investigator's involvement in this study is unlikely to be bias-free. Researchers actively conducted the 12-week intervention and developed friendships with participants. As the research team wanted the intervention to succeed, survey questions may have been framed in a more positive light. Respondents offered little criticism about the intervention, likely to maintain social desirability. If an independent researcher had conducted the surveys, the results may have been different.

Participants saw the researchers as a source of knowledge to guide them on their journey of behaviour change with regard to physical activity and well-being. This dynamic made certain aspects of the survey process challenging as some of the respondents felt that they were 'not qualified' enough to answer the questions and criticise aspects of the intervention. The dynamic between researcher and participant did, however, contribute to the success of the intervention. The participants did not consider the researcher as superior to them and actively engaged with them on an individual basis. This type of attention is key to the success of a lifestyle intervention. ${ }^{[3]}$

\section{Practical application}

To date, the profession of biokinetics has been confined to the private health sector. A major component of a biokineticist's training involves using exercise modalities in the prevention and treatment of NCDs, such as obesity, type 2 diabetes and heart disease. These skills are urgently required within the public health sector to slow the devastating impact of NCDs on this country's communities and economy.

Many biokineticists are denied internship positions due to the lack of capacity within the private health sector. The utilisation of both intern and qualified biokineticists in the public health sector provides a cost-effective approach to combat an increasingly sedentary South African lifestyle. The lifestyle intervention model used in this study is easily reproducible and demonstrates the potential for a wide array of positive effects on its participants; hence future research should expand upon this.

\section{Study limitations}

It is important to note that this intervention was a pilot study, the main purpose of which was to facilitate future research. Although the study demonstrated favourable results, the inferences that can be made with the small sample size, lack of a control group and predominately self-report measures are limited. The Sweet Hearts programme was organised and conducted by a single postgraduate student. No funding for the running costs of the intervention was received, which mirrors somewhat the lack of financial support and human capital available in combating the rise of NCDs through physical activity and health education in South Africa.

The aim of the qualitative component of the study was to understand the social dynamics of the intervention, not generalisability. The researchers used a purposive sampling technique to recruit respondents, thus bias was introduced, as not all participants could be contacted and surveyed. The researchers acknowledge that their findings did not represent the views of participants who were not interviewed. Social desirability may have also resulted in respondents favouring positive feedback whilst avoiding that which criticised the intervention.

\section{Conclusion}

This pilot study examined the effect of a 12-week biokinetics primary prevention programme on health outcome measures. A total of 27 exercise sessions, combined with nutritional and healthy lifestyle advice, were conducted. The intervention experienced a high attrition rate with $>50 \%$ of participants not presenting for post-intervention testing. Despite this limitation and the small sample size $(n=10)$, the intervention showed significant improvements in health outcome measures amongst participants ( $p \leq 0.05)$. These improvements included: an increased Global Physical Activity Questionnaire (GPAQ) score, a 12-minute walk distance, sit-to-stand test repetitions, and a decrease in waist circumference. Improvements were also noted in cognitive restraint with regards to eating behaviour. The qualitative surveys conducted $(n=5)$ offer valuable insight into the issue of adherence and how to improve the Sweet Hearts intervention. Potential improvements include the use of an indoor venue, an increase from two to three sessions per week, inclusion of midway assessments, more detailed dietary guidance and greater diversity in the demographics of participants. The results of the Sweet Hearts intervention demonstrate the difficulty and importance of maintaining adherence to a community health intervention programme. The favourable results of the small sample size demonstrate the potential benefit of biokinetics-based programmes in the public health sector; and provide proof of concept for the dedication of resources towards health promotion within a community setting.

Acknowledgements: Scholarships for the postgraduate student who conducted this study were received from the University of Cape Town (UCT) and the National Research Foundation (NRF). The authors wish to thank the Biokinetic Humanitarian Project (BHP), Victoria Hospital Wynberg, Tramway Football Club, and biokineticists Devon Coetzee, Samantha Jones and Megan Grobler for their assistance in conducting this project.

\section{References}

1. Bradshaw D, Norman R, Pieterse D, et al. Estimating the burden of disease attributable to diabetes in South Africa in 2000. S Afr Med J 2007;97 (8Pt2): 700-706. [PMID:1795227]]

2. Strategic Plan for the Prevention and Control of NonCommunicable Diseases 2013-17. 2013. Available from: http://www.hsrc.ac.za/uploads/pageContent/3893/NCDs STRAT PLAN CONTENT 8 april proof.pdf.RP06/2013. ISBN: 978-0-621-4151-0.

3. Marcus BH, Williams DM, Dubbert PM, et al. Physical activity intervention studies: what we know and what we need to know: a scientific statement from the American Heart Association Council on Nutrition, Physical Activity, and Metabolism (Subcommittee on Physical Activity); Council on Cardiovascular Disease in the Young; and the Interdisciplinary Working Group on Quality of Care and Outcomes Research. 
Circulation 2006;114(24):2739-2752.

[DOI:. 10.1161/CIRCULATIONAHA.106.179683]

4. Scarinci IC, Moore A, Wynn-Wallace T,et al. A communitybased, culturally relevant intervention to promote healthy eating and physical activity among middle-aged African American women in rural Alabama: findings from a group randomized controlled trial. Prev Med 2014;69:13-20. [DOI: 10.1016/j.ypmed.2014.08.016]

5. Fleury J, Lee SM. The social ecological model and physical activity in African American women. Am J Community Psychol 2006;37:129-140. [DOI: 10.1007/s10464-005-9002-7]

6. Evans RW, Smith T, Kay P, et al. The need for biokineticists in the South African public health care system. S Afr J Sports Med 2016;28:85-86. [DOI. 10.17159/2078516x/2016/v28i3a1310]

7. Sampson L. A multidisciplinary approach to cardiac rehabilitation in SA. S Afr Med J 2014;104:94. [PMID: 24893527]

8. American College of Sports Medicine. ACSM's Guidelines for Exercise Testing and Prescription, 7th ed, Baltimore, MD: Lippincott Williams \& Wilkins, 2006.

9. Noorbhai MH. A public health approach to increase physical activity and health education: The Biokinetic Humanitarian Project. Afr J Phys Heal Educ Recreat Danc 2013;19:993-998.
10. de Lauzon B, Romon M, Deschamps V, et al. The Three-Factor Eating Questionnaire-R18 is able to distinguish among different eating patterns in a general population. J Nutr 2004;134:2372-2380. [PMID:15333731]

11. Cooper KH. A means of assessing maximal oxygen intake: Corrolation between field and treadmill testing. JAMA 1968;203(3):201-204. [PMID: 5694044]

12. Koster A, Leitzmann MF, Schatzkin A, et al. Waist circumference and mortality. Am J Epidemiol 2008;167:14651475. [.DOI:10.1093/aje/kwn079]

13. Mccready ML, Long BC. Locus of Control, Attitudes Toward Physical Activity, and Exercise Adherence. J Sport Psychol 1985;7:346-359. [https:// doi.org/10.1123/jsp.7.4.346]

14. White JL, Ransdell LB, Vener J, et al. Factors related to physical activity adherence in women: review and suggestions for future research. Women Health 2005;41:123-148. [DOI:10.1300/J013v41n04 07]

15. Cobb-Clark DA, Kassenboehmer SC, Schurer S. Healthy habits: The connection between diet, exercise, and locus of control. J Econ Behav Organ 2014;98:1-28. [doi.org/10. 1016/j.jebo.2013.10.011]

16. Thomas SG, Goodman JM, Burr JF. Evidence-based risk assessment and recommendations for physical activity clearance: established cardiovascular disease. Appl Physiol Nutr Metab 2011;36:S190-213. [DOI: 10.1139/h11-050] 